\title{
Leprosy elimination campaign (LEC) in the Philippines
}

\author{
JOSE BENITO R. VILLARAMA \\ Coordinator, National Leprosy Control Program, Philippines
}

Accepted for publication 1 September 1999

\section{Introduction}

A leprosy elimination campaign (LEC) was implemented in the Philippines as a means to intensify case-finding activities, in view of the perceived large gap between estimated and actual new cases detected. The objective was to find all 'hidden cases' of leprosy, particularly cases of consequence, and treat them with multi-drug therapy (MDT).

LEC projects in the Philippines were first introduced in 1996 in two provinces, namely Ilocos Norte and Cebu. Thereafter, 13 others were implemented, four in 1997 (Ilocos Sur, Maguindanao, Cagayan, Cebu City) and nine in 1998, of which seven were completed in 1998 (Pangasinan, La Union, Rizal, Antique, Zamboanga Norte, Misamis Oriental, Sarangani) and two in 1999 (Davao Sur, Biliran).

The results of LEC projects are very encouraging. Thus, the projects narrowed the perceived gap, particularly in areas where the number of suspected backlog cases was high.

\section{Materials and methods}

The standard protocol of activities undertaken in the implementation of LEC projects in the Philippines include the following:

CO-ORDINATION WITH LOCAL GOVERNMENT EXECUTIVES (LGE)

Local Government Executives (LGEs) are informed about the purpose and expected outcome of the project. Commitments from LGEs is obtained through a Memorandum of Agreement between the LGEs, provincial and regional health office, and National Leprosy Control Programme, specifying duties and responsibilities of each in the implementation of the project.

FORMATION OF LEC TEAMS

At least three LEC teams are organized per province. Each LEC team is headed by a doctor, 
nurse, or a nursing attendant who has been trained to diagnose leprosy. The members of the LEC team are representatives from the Regional and Provincial Health Office, Sanitarium, Skin Clinic, and Rural Health Unit The LEC teams conduct skin consultation (based on a masterlist of persons with skin diseases) in a designated area in every barangay in a municipality on a scheduled date.

\section{CAPABILITY BUILDING}

LEC team leaders are oriented on the LEC project and its implementation. A 1-day refresher training course on leprosy is also provided to enhance ability to diagnose and appropriately manage leprosy and its complications. Schedule of LEC team visit per municipality is drawn during this orientation training.

The Municipal Health Officers, who are also the LEC team leaders in their respective municipality, are then tasked to orient their staff community volunteer health workers (CVHW) and village leaders on the project. The date of LEC team visit per barangay is scheduled during this half-day orientation.

\section{PROCUREMENT OF TOPICAL OINTMENTS}

Procurement of topical ointments (antibiotic, anti-fungal and anti-scabies) is based on the expected number of skin consultations. These ointments, prepared by the sanitarium, serve as an incentive for individuals with skin lesions to consult with the LEC team.

\section{COMMUNITY PREPARATION}

One to 2 weeks before the scheduled LEC team visit, CVHW prepare the community by informing every household that a team of skin experts (LEC team) will visit the barangay on a scheduled date, time, and place. CVHW list persons with skin lesions in a master list and encourage them to seek skin consultation with the LEC team for management of skin lesions.

Community assemblies are also done to inform the community of the importance of skin consciousness and provide correct information that leprosy is curable with multi-drug therapy (MDT).

\section{LAUNCHING OF LEC}

The start of LEC activities is usually ushered in by a motorcade in the provincial capital or municipality with the highest leprosy prevalence. Before and during the launch, intensive information dissemination campaign using various forms of media (TV/radio interviews, press releases, display of leprosy posters, and distribution of leaflets) is undertaken to increase the level of public awareness on leprosy. This serves to remind individuals with skin lesions to consult with the visiting LEC team.

\section{CASE FINDING AND TREATMENT}

Each LEC team is expected to cover at least two barangays per day. All masterlisted individuals are screened out for leprosy and given appropriate treatment. Individuals who failed to come for consultation are followed up by the CVHW or rural health unit staff. 
ENDORSEMENT OF DIAGNOSED LEPROSY CASES TO RHU

The patient's record form is filled up for every new diagnosed leprosy case and endorsed to the respective Rural Health Unit (RHU) for follow-up and further management. Names of new cases are added into the existing Central Registry and Drug Collection Chart.

\section{Results}

Fifteen LEC projects have been implemented in the Philippines from 1996 to April 1999, covering $12,412,063(17 \%)$ of the total population or $29 \%$ of the target population, that is, from provinces with a PR of $>1 / 10,000$ population in 1997 . These projects detected a total of 1408 new cases. Fifty-nine percent (825) of these new cases were of multibacillary (MB) leprosy, 9\% (121) were grade 2 deformity and 13\% (179) were less than 15 years old (Table 1).

\section{Discussion}

Eleven (Ilocos Norte, Cebu, Cagayan, Maguindanao, Ilocos Sur, Pangasinan, Rizal, Zamboanga Norte, Antique, Biliran, Davao Sur) of the 15 LEC provinces had a 4-218\% increase in new cases detected compared to routine case finding activities (Figure 1). Ilocos Sur showed the highest increase, with 207 new cases detected in the 1997 LEC compared to only 65 new cases for the whole year of 1996. LEC in Cebu Province in 1996 found 154 new cases, only a 4\% increase from its 148 new cases in 1995.

LEC for Cebu City detected only 46 new cases in 1997 compared to 80 new cases from routine case finding activities in 1996 . This could be attributed to:

Table 1. Profile of new leprosy cases in 15 LEC areas, Philippines, 1996-1999

\begin{tabular}{|c|c|c|c|c|c|}
\hline Province & Population & $\begin{array}{c}\text { New cases } \\
\text { detected }\end{array}$ & MB cases & $\begin{array}{c}\text { With grade } 2 \\
\text { deformity }\end{array}$ & $<15$ years old \\
\hline Ilocos Norte & 501,872 & 97 & 53 & 23 & 11 \\
\hline Cebu & $1,416,301$ & 154 & 108 & 19 & 35 \\
\hline Cagayan & 915,154 & 105 & 52 & 15 & 15 \\
\hline Maguindanao & 674,420 & 210 & 89 & 22 & 36 \\
\hline Ilocos Sur & 601,421 & 207 & 65 & 5 & 20 \\
\hline Cebu City & 691,623 & 46 & 30 & 3 & 12 \\
\hline La Union & 626,766 & 41 & 25 & 4 & 3 \\
\hline Pangasinan & $2,269,244$ & 130 & 107 & 8 & 12 \\
\hline Rizal & $1,549,081$ & 58 & 34 & 2 & 3 \\
\hline Zamboanga & 825,726 & 112 & 78 & 1 & 14 \\
\hline \multicolumn{6}{|l|}{ Norte } \\
\hline Sarangani & 424,358 & 25 & 23 & 1 & 1 \\
\hline Antique & 446,647 & 86 & 62 & 7 & 6 \\
\hline Misamis & 622,951 & 32 & 19 & 3 & 1 \\
\hline Oriental & & & & & \\
\hline Biliran & 140,921 & 28 & 19 & 2 & 4 \\
\hline Davao Sur & 705,578 & 77 & 61 & 6 & 6 \\
\hline Total & $12,412,063$ & 1,408 & $\begin{array}{l}825 \\
(59 \%)\end{array}$ & $\begin{array}{l}121 \\
(9 \%)\end{array}$ & $\begin{array}{l}179 \\
(13 \%)\end{array}$ \\
\hline
\end{tabular}




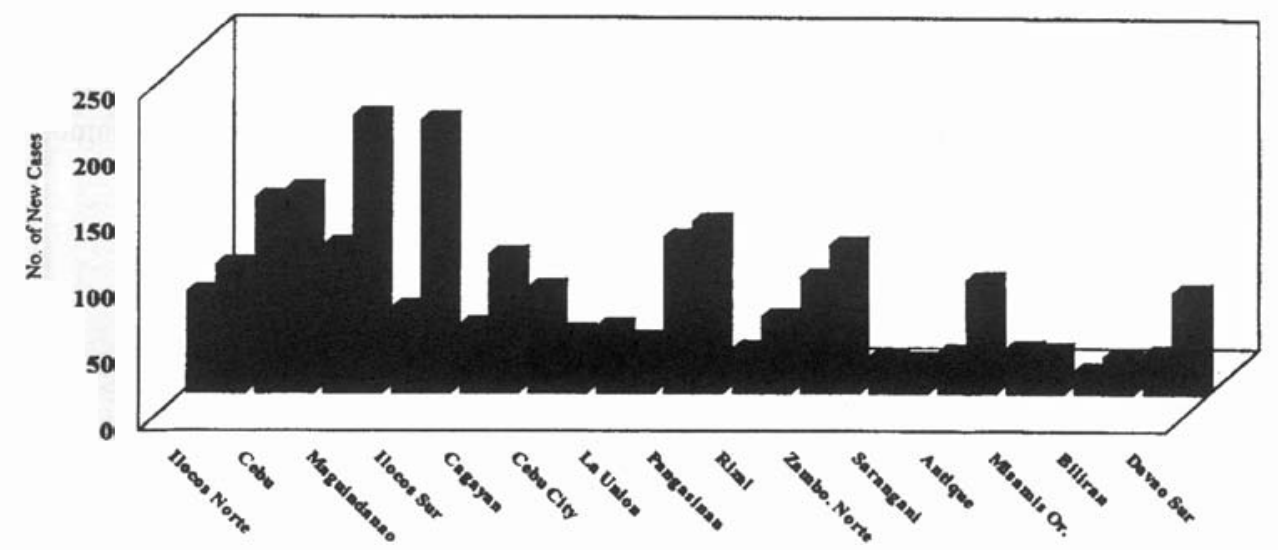

Figure 1. Case detection: routine (first bar in each pair) versus LEC (second bar in each pair).

1. Few new cases truly resident in Cebu City. Some cases detected during routine case finding are actually from surrounding municipalities because of fear of being ostracized in their municipalities and/or belief that city's leprosy services are of better quality.

2. The target population work on weekdays or are too busy attending to personal matters. Thus, it is also advisable to carry out LEC activity on weekends.

La Union detected only 41 new cases during its 1998 LEC, compared to 50 new cases the previous year. This is due to mini-LECs (municipal wide) done prior to the implementation of the LEC (province wide). The provinces of Sarangani and Misamis Oriental had satisfactory LEC activity even though the number of new cases detected was almost the same as its routine case finding because of the shorter detection time frame, 3 months compared to 1 year.

LEC MB cases accounts for $62 \%$ of all MB cases detected in the LEC areas during the same year when LEC was done. The proportion of MB cases among new cases reached 59\%



Figure 2. 1996 and 1997 LEC accomplishments. First bar in each group represents new cases, second bar represents MB cases, third bar represents number with grade 2 disability and fourth bar represents those under 15 years of age. 


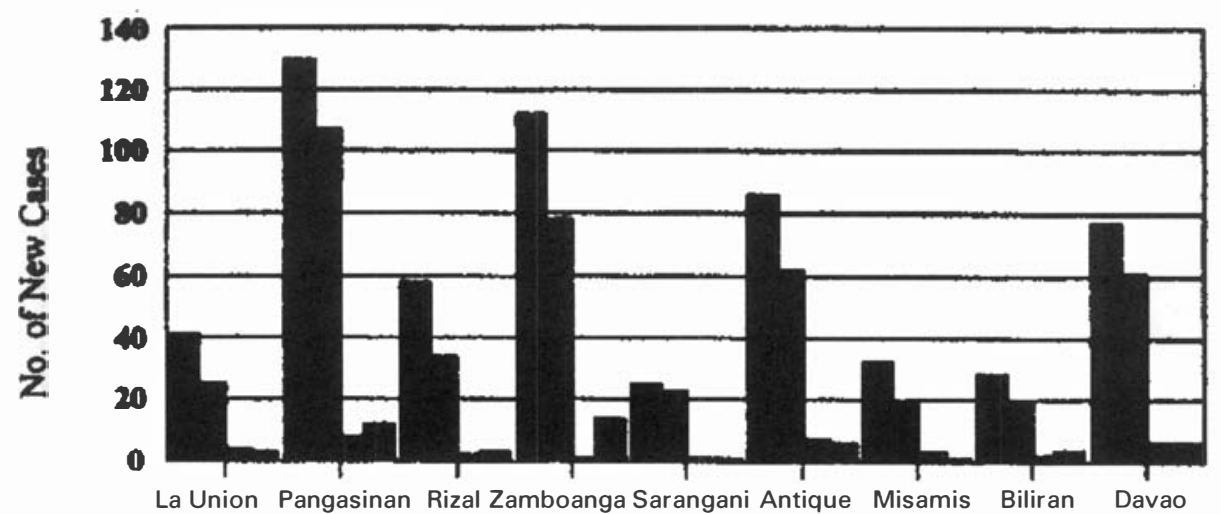

Figure 3. 1998 and 1999 LEC accomplishments. First bar in each group represents new cases, second bar represents MB cases, third bar represents number with grade 2 disability and fourth bar represents those under 15 years of age.

during LEC, in contrast to $80 \%$ with routine case finding. Further, the proportion of new cases with grade 2 deformity significantly increased to $9 \%$ in LEC areas, compared to the annual average of 5\%. Eighty-nine percent of the new cases with grade 2 deformity were found through LEC in LEC provinces. The MB cases, particularly those with grade 2 deformity, represent backlog cases. Figures 2 and 3 show the LEC accomplishments for respective LEC provinces.

A declining trend in the number of new cases is seen in the 1996 and 1997 LEC areas (Figure 4). However, the proportion of MB cases among new cases 1-2 years after LEC has not changed from $79 \%$ to $89 \%$ (Figure 5). Further, the proportion of new cases with grade 2 deformity has not gone down except in Cebu Province, from 6\% (1 year after LEC) to $4 \%$ (2 years after LEC) (Figure 6). The proportion of new cases $<15$ years old has decreased in LEC areas, as shown in Figure 7, which could reflect a probable reduction in the transmission of the disease in the community.

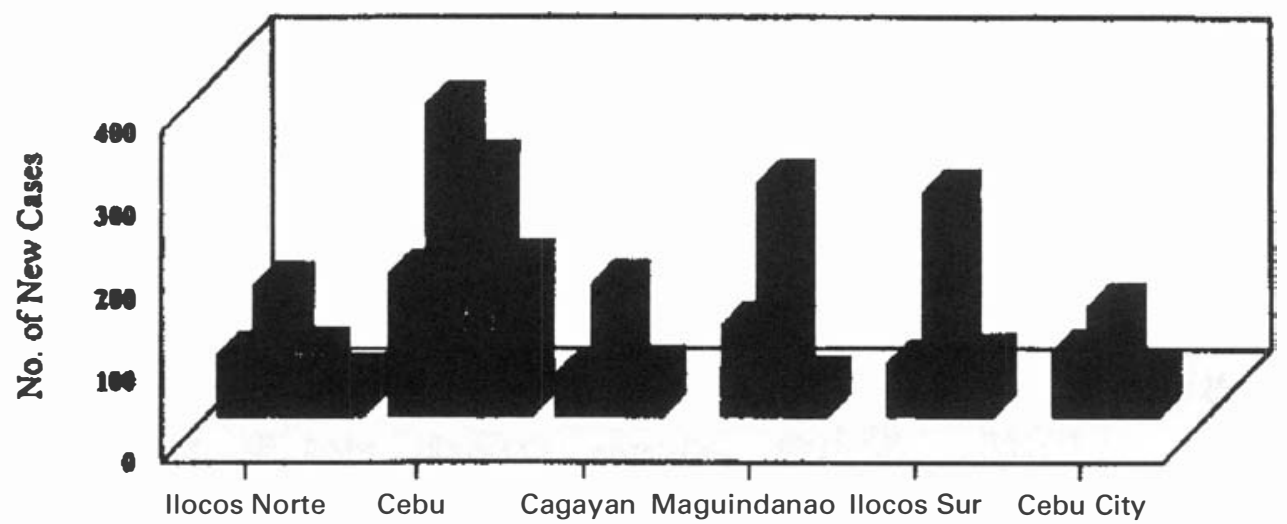

Figure 4. New cases detected 1995-1998, 1996 and 1997 LEC areas, Philippines. First bar in each group represents year before LEC, second bar represents LEC, third and fourth bars represent 1 and 2 years after LEC, respectively. 


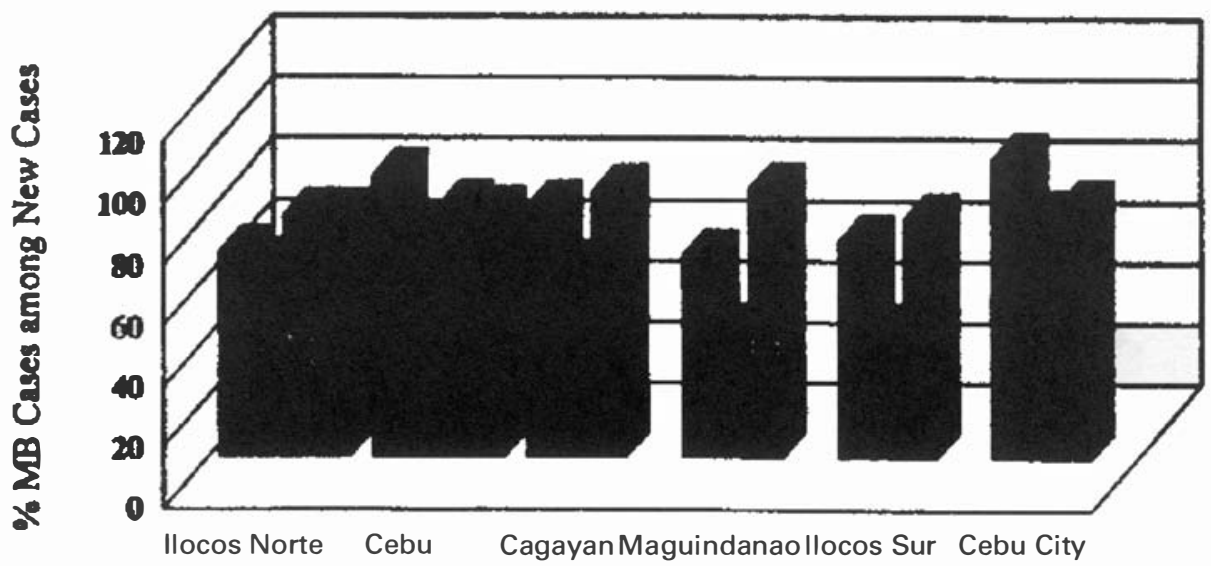

Figure 5. Percentage MB cases among new cases, 1996 and 1997 LEC areas, Philippines. First bar in each group represents year before LEC, second bar represents LEC, third and fourth bars represent 1 and 2 years after LEC, respectively.

A total of $\$ 112,862$ was spent for the 15 LEC projects, giving an average cost of $\$ 80 \cdot 16$ per new leprosy case detected.

It is worth noting that there are LEC provinces with poor and good accomplishments. The contributory factors that have brought about failure or success of LEC activities in the Philippines are as follows:

\section{FACTORS CONTRIBUTING TO POOR LEC OUTPUT}

1. Poor LGE support in some municipalities:

- No available transport.

- Late arrival of LEC team.

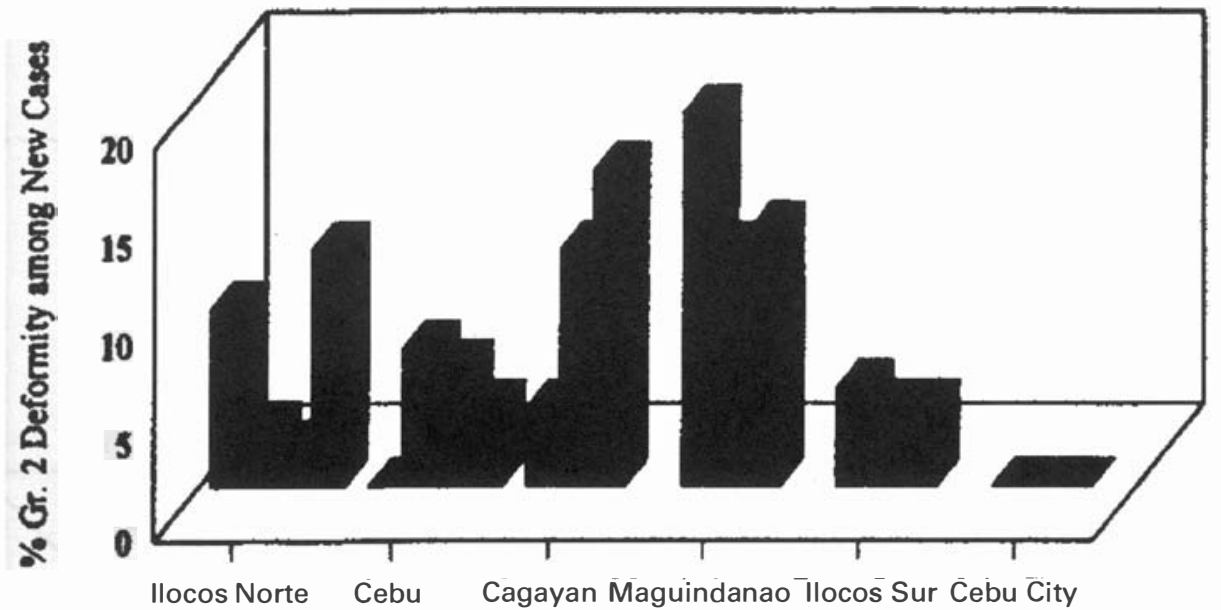

Figure 6. Percentage grade 2 deformity among new cases, 1996 and 1997 LEC areas, Philippines. First bar in each group represents year before LEC, second bar represents LEC, third and fourth bars represent 1 and 2 years after LEC, respectively. 


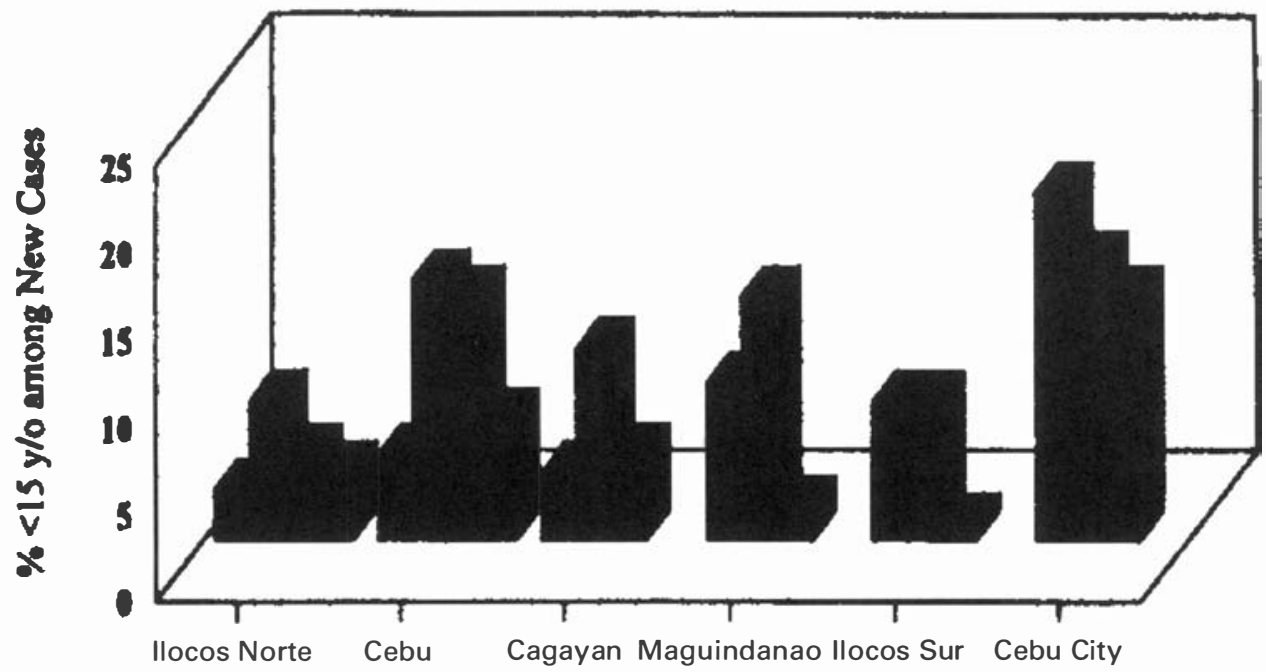

Figure 7. Percentage under 15 years old among new cases, 1996 and 1997 LEC areas, Philippines. First bar in each group represents year before LEC, second bar represents LEC, third and fourth bars represent 1 and 2 years after LEC, respectively.

2. Poor co-ordination of project activities.

3. Wrong timing of implementation:

- Coincided with:

election campaign.

other priority DOH activities.

- Rainy season.

4. Inaccessibility of some barangays.

FACTORS CONTRIBUTING TO GOOD LEC OUTPUT

1. Health oriented LGEs.

2. Committed coordinators.

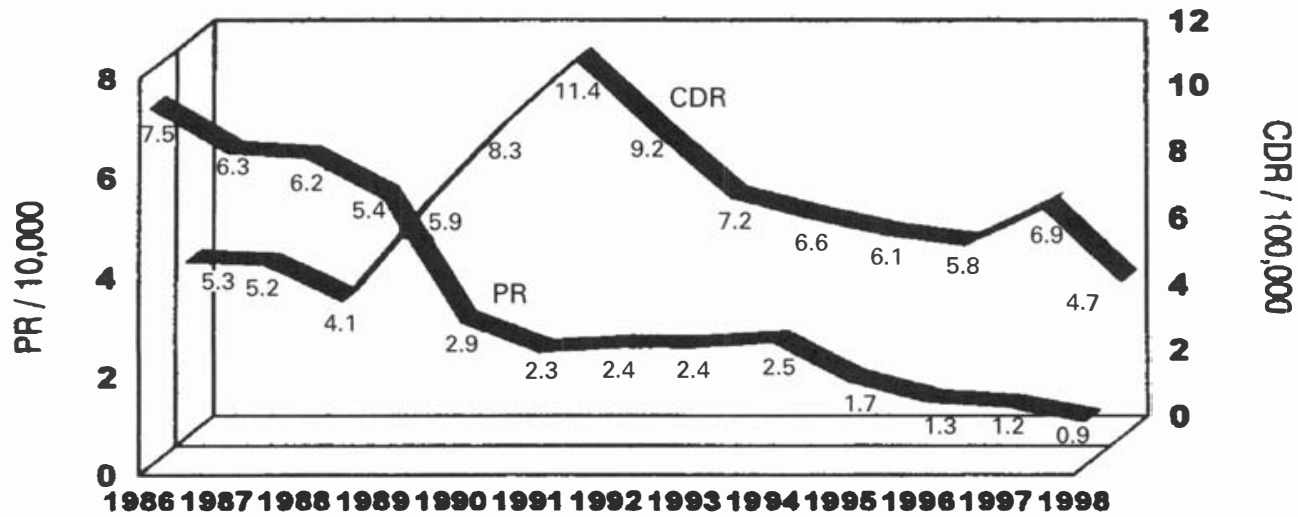

Figure 8. Prevalence and case detection rate, 1986-1998, Philippines. 
3. Dedicated field health workers.

4. Availability of logistic support.

To sustain LEC activities initiated in LEC areas, the following have to be undertaken:

1. Ensure continuance of LGE support.

2. Undertake regular monitoring and supervision of coordinators.

3. Conduct local programme assessment by NLCP staff.

4. Hold consultative meetings with coordinators.

5. Provide logistic support for Kilatis Kutis Campaign which is highlighted during Annual Leprosy Control Week.

The implementation of LEC in the Philippines increased the case detection rate (CDR) from $5 \cdot 8 / 100,000$ population (4051 new cases) in 1996 to $6 \cdot 9 / 100,000$ population (4942 new cases) in 1997 or a $22 \%$ increase in the number of new cases. However, the prevalence rate (PR) continued to decrease from $1 \cdot 3 / 10,000$ population in 1996 to $1 \cdot 2 / 10,000$ population in 1997. Despite the implementation of more LEC projects in 1998, the PR and CDR still went down to $0 \cdot 9 / 10,000$ population and $4 \cdot 7 / 100,000$ population, respectively. The steady decline in PR is attributed to the implementation of the shorter treatment course and updating of records. It is projected that the PR and the CDR will continue to decline even if special case finding projects are implemented (Figure 8).

In conclusion, LEC, when properly co-ordinated, yields best results. Indeed, LEC is a very effective means of detecting backlog cases, thus reducing the gap between estimated and actual new cases detected. However, activities initiated by LEC should be sustained, to make the project more cost effective and cost efficient. 